\section{BIOTECH STOCKS}

NEW YORK - The boom is back in biotech. Steven Rosenberg's work at the National Cancer Institute, which demonstrated preliminary success using interleukin- 2 on 30 cancer patients, made the evening news. November 25's issue of Fortune proclaimed that researchers "have now discovered how to use a small group of substances produced by the body's own immune system to control all cancers." And, as the Dow Jones industrial average attained the 1500 mark, biotech stocks have soared.

The Bio/Technology Index of Specialty Firms climbed almost 30 percent in the four weeks preceding December 9 , reaching a record high of 1535. During that period, three large gainers were the companies highlighted in Fortune: Genentech stock gained 27 percent to $\$ 70$ per share, Immunex advanced 70 percent to $\$ 14$, and Cetus shot up 78 percent to about $\$ 30$. (Genentech's increase was fueled additionally by continued ru-

SURGE TO RECORD HIGHS

mors of it as a potential takeover target. Its market value now approaches $\$ 1.2$ billion, however, making it quite a large bite to swallow.) Among the monoclonal specialists, the companies awaiting final approvals on their acquisitions-Hybritech and Genetic Systems-moved very little. Centocor, by comparison, gained 25 percent to almost $\$ 27$.

Analysts now face the task of determining whether the upward trend will continue, or if the down-side of the roller coaster lies just around the next bend. Peter F. Drake, biomedical technology analyst for Kidder, Peabody (New York, NY), notes that the last two years have seen January rallies in biotechnology stocks. If this pattern repeats, he says, 1986 could prove a big year. If not, he predicts, the sector could be in for a pull-back.

Linda Miller of Paine Webber (New York, NY) says she is "very optimistic" for 1986, as she looks for new products, continued mergers and ac- quisitions, and increased investment on the part of large companies. She predicts that the public market may open up again, so 1986 could see some of the few remaining private concerns making public offerings.

"I think 1986 might be the year when we start seeing through the smoke," and start getting meaningful clinical data on tissue-type plasminogen activator, gamma interferon, and the interleukins, says Steven Zimmer, vice president of Eberstadt Fleming (New York, NY). He notes, however, that many stock prices doubled in 1985 , so they may currently be overvalued. "We're getting into dangerous territory now," he concludes.

"My hunch is that the biotechnology stocks will underperform the market in 1986," concurs Eugene J. Rothman, health care analyst for Alex. Brown (Boston, MA). "They may still go up," he adds, "but stocks in other industries may go up more."

-Arthur Klausner

\title{
STOCK PRICES OF SELECTED BIOTECHNOLOGY SPECLAITY FIRMS
}

\begin{tabular}{|c|c|c|c|c|c|}
\hline & $\begin{array}{c}\text { Price } \\
\text { July } 9,1985\end{array}$ & $\begin{array}{c}\text { Price } \\
\text { Dec. } 9,1985\end{array}$ & $\begin{array}{l}\text { Percent } \\
\text { Change }\end{array}$ & $\begin{array}{l}52 \text { Week } \\
\text { Hi-Low }\end{array}$ & $\begin{array}{l}\text { Market Capitalization } \\
\text { in } \$ \text { millions (stock } \\
\text { price } \times \text { number of } \\
\text { shares outstanding) }\end{array}$ \\
\hline \multicolumn{6}{|c|}{$\begin{array}{l}\text { Companies Emphasizing Recombinant } \\
\text { DNA Technology }\end{array}$} \\
\hline Advanced Genetic Sciences & $37 / 8$ & $47 / 8$ & +26 & $73 / 4-21 / 2$ & 54 \\
\hline *Amgen & $73 / 8$ & $127 / 8$ & +75 & $153 / 4-41 / 4$ & 139 \\
\hline *Bio Logicals & $15 / 16$ & $13 / 16$ & -10 & $21 / 8-15 / 16$ & 7 \\
\hline *Biogen & $7 \%$ & $151 / 8$ & +98 & $18 \% / 8-43 / 4$ & 281 \\
\hline *Biotech Research Labs & $67 / 8$ & $91 / 4$ & +35 & $101 / 2-6$ & 49 \\
\hline *Biotechnica International & $81 / 4$ & $93 / 4$ & +18 & $101 / 2-61 / 4$ & 41 \\
\hline Biotechnology General & $71 / 2$ & $81 / 2$ & +13 & $83 / 4-33 / 4$ & 39 \\
\hline California Biotechnology & $11 \% / 4$ & $14 \frac{1}{4}$ & +21 & $15-91 / 2$ & 93 \\
\hline *Cetus & $10 \% / 4$ & $293 / 8$ & +173 & $333 / 8-85 / 8$ & 673 \\
\hline Chiron & $73 / 4$ & $121 / 8$ & +56 & $151 / 8-43 / 8$ & 89 \\
\hline *Collaborative Research & 4 & $53 / 4$ & +44 & $71 / 8-23 / 4$ & 58 \\
\hline CooperBiomedical & $23 / 16$ & $1^{13 / 16}$ & -17 & $55 / 8-15 / 16$ & 32 \\
\hline *Enzo Biochem & $111 / 8$ & $143 / 4$ & +33 & $211 / 4-10$ & 131 \\
\hline${ }^{*}$ Genentech & 46 & $701 / 4$ & +53 & $75^{1 / 4}-31^{1 / 2}$ & 1187 \\
\hline *Genex & $27 / 8$ & $23 / 16$ & -24 & $73 / 4-13 / 8$ & 28 \\
\hline Integrated Genetics & $33 / 4$ & $51 / 8$ & +37 & $61 / 8-3$ & 43 \\
\hline *Molecular Genetics & $61 / 2$ & $11 \frac{1}{4}$ & +73 & $13-61 / 8$ & 70 \\
\hline \multicolumn{6}{|l|}{$\begin{array}{l}\text { Companies Emphasizing Antibody } \\
\text { Production Technologies }\end{array}$} \\
\hline $\begin{array}{l}\text { Production Technologies } \\
* \text { Bio-Response }\end{array}$ & $67 / 8$ & $8^{1 / 8}$ & +18 & $93 / 8-33 / 4$ & 66 \\
\hline *Cambridge BioScience & $41 / 8$ & 5 & +21 & $5 \% / 8-11 / 8$ & 21 \\
\hline *Centocor & $173 / 4$ & $267 / 8$ & +51 & $267 / 8-8$ & 194 \\
\hline *Damon Biotech & $6^{1 / 2}$ & 9 & +38 & $91 / 4-41 / 8$ & 174 \\
\hline *Genetic Systems & 7 & 10 & +43 & $10-5$ & 198 \\
\hline${ }^{*}$ Hybritech & 25 & $291 / 2$ & +18 & $30^{1 / 8}-14$ & 316 \\
\hline *Monoclonal Antibodies & 9 & 18 & +100 & $191 / 4-79 / 4$ & 43 \\
\hline *Summa Medical & $31 / 8$ & $23 / 4$ & -12 & $43 / 4-15 / 8$ & 38 \\
\hline \multicolumn{6}{|l|}{$\begin{array}{l}\text { Companies Emphasizing Other } \\
\text { Products or Biotechnologies }\end{array}$} \\
\hline $\begin{array}{l}\text { Products or Brotechnologies } \\
\text { Applied Biosystems }\end{array}$ & 23 & 30 & +30 & $30-81 / 4$ & 339 \\
\hline *Genetic Engineering Inc. & $21 / 8$ & $11 / 16$ & -50 & $4-1 / 2$ & 3 \\
\hline *Immunex & $53 / 4$ & 14 & +143 & $163 / 4-4$ & 83 \\
\hline *Interferon Sciences & $35 / 8$ & $53 / 4$ & +59 & $61 / 2-21 / 2$ & 23 \\
\hline *Ribi Immunochem & 8 & $75 / 8$ & -5 & $10-33 / 8$ & 26 \\
\hline Vega Biotechnologies & $17 / 8$ & $1^{15 / 16}$ & +3 & $33 / 8-11 / 8$ & 5 \\
\hline
\end{tabular}

